\title{
The dopamine imbalance hypothesis of fatigue in multiple sclerosis and other neurological disorders
}

\author{
Ekaterina Dobryakova ${ }^{1,2}$ *, Helen M. Genova ${ }^{2,3}$, John DeLuca ${ }^{2,3,4}$ and Glenn R. Wylie ${ }^{2,3,5}$ \\ ${ }^{1}$ Traumatic Brain Injury Laboratory, Kessler Foundation, West Orange, NJ, USA \\ ${ }^{2}$ Department of Physical Medicine and Rehabilitation, Rutgers - New Jersey Medical School, Newark, NJ, USA \\ ${ }^{3}$ Neuropsychology and Neuroscience Laboratory, Kessler Foundation, West Orange, NJ, USA \\ ${ }^{4}$ Department of Neurology and Neurosciences, Rutgers - New Jersey Medical School, Newark, NJ, USA \\ ${ }^{5}$ War Related IIIness and Injury Study Center, Department of Veterans Affairs, East Orange, NJ, USA
}

\section{Edited by:}

Christian Dettmers, Kliniken

Schmieder Konstanz, Germany

\section{Reviewed by:}

Maria Engström, Linköping

University, Sweden

Olivia Marie Reinhold Svolgaard,

Copenhagen University Hospital

Hvidovre, Denmark

\section{${ }^{*}$ Correspondence}

Ekaterina Dobryakova, Traumatic Brain Injury Laboratory, Kessler Foundation, 1199 Pleasant Valley Way,

West Orange, NJ 07052, USA

e-mail:edobryakokva@

kesslerfoudnation.org
Fatigue is one of the most pervasive symptoms of multiple sclerosis (MS), and has engendered hundreds of investigations on the topic. While there is a growing literature using various methods to study fatigue, a unified theory of fatigue in MS is yet to emerge. In the current review, we synthesize findings from neuroimaging, pharmacological, neuropsychological, and immunological studies of fatigue in MS, which point to a specific hypothesis of fatigue in MS: the dopamine imbalance hypothesis. The communication between the striatum and prefrontal cortex is reliant on dopamine, a modulatory neurotransmitter. Neuroimaging findings suggest that fatigue results from the disruption of communication between these regions. Supporting the dopamine imbalance hypothesis, structural and functional neuroimaging studies show abnormalities in the frontal and striatal regions that are heavily innervated by dopamine neurons. Further, dopaminergic psychostimulant medication has been shown to alleviate fatigue in individuals with traumatic brain injury, chronic fatigue syndrome, and in cancer patients, also indicating that dopamine might play an important role in fatigue perception. This paper reviews the structural and functional neuroimaging evidence as well as pharmacological studies that suggest that dopamine plays a critical role in the phenomenon of fatigue. We conclude with how specific aspects of the dopamine imbalance hypothesis can be tested in future research.

Keywords: dopamine, fatigue, mesocorticolimbic system, methylphenidate, MRI

\section{INTRODUCTION}

Fatigue is a common symptom in multiple sclerosis (MS), with up to $90 \%$ of individuals with MS reporting fatigue (1). Moreover, more than half of individuals with MS report fatigue to be their worst symptom (2). For this reason, the topic of fatigue in MS has generated a great deal of research in the domains of neuropsychology, neuroscience, and pharmacology. Other clinical populations also report fatigue including: $80 \%$ of individuals with traumatic brain injury (TBI) (3), $56 \%$ of individuals with Parkinson's Disease (PD) (4), 99\% of cancer patients (5), $88 \%$ of individuals with human immunodeficiency virus (88\%) (6), as well as individuals with chronic fatigue syndrome (CFS) who experience fatigue for more than 6 months for no known psychiatric or neurological reasons ( 7 ).

Fatigue is characterized by a lack of energy, feelings of exhaustion that are unaided by sleep, and the perception that one is unable to perform mental and physical activities (8). Although fatigue has been studied extensively, in part because it affects such a wide range of clinical populations, there has been no unifying framework within which to understand fatigue. In this review, we propose such a framework, with the aim of providing structure for this developing field of study.

We propose that fatigue arises due to a dopamine imbalance within the central nervous system (CNS). One of the ultimate goals of this review is to investigate the evidence that supports the dopamine imbalance hypothesis by examining studies showing structural and functional abnormalities in areas enervated by dopamine and clinical trials showing alleviation of fatigue after dopamine medication.

The current review examines the evidence in support of the dopamine imbalance hypothesis by focusing on central fatigue, which can be experienced as both physical and mental in nature. Further, the current review builds upon a previous framework of fatigue proposed by Chaudhuri and Behan (9), which suggests that central fatigue might arise due to the "failure of the non-motor functions of the basal ganglia" [(9), p. 40]. This hypothesis was developed based on evidence from both animal and clinical studies, which showed the effects of basal ganglia damage to be similar to the symptoms of central fatigue. The authors emphasized subcortical pallido-thalamo-cortical interactions and urged to clarify the influence of dopamine and serotonin on fatigue, since these neurotransmitters effect the activation of the pallido-thalamocortical loop. In the current review, we suggest a more precise mechanism based on recent studies that fatigue might develop as a result of a dopamine imbalance.

\section{DOPAMINE: A BRIEF REVIEW}

Dopamine is a modulatory neurotransmitter that is termed a catecholamine due to its chemical composition. Dopamine is the most common catecholamine in the CNS $(10,11)$ and is a precursor 
to the two other catecholamines, norepinephrine and epinephrine. In the CNS, dopamine is synthesized in two subcortical brain regions, specifically, the substantia nigra pars compact $(\mathrm{SNc})$ and the ventral tegmental area (VTA) $(10,12-14)$. Dopaminergic neurons project from the SNc and VTA to various cortical areas and thus can be segregated onto several dopaminergic pathways: (1) the nigrostriatal pathway, which links the SNc with the striatum, and (2) the mesocorticolimbic pathway, which starts at the VTA and projects to the striatum, limbic areas, and the prefrontal cortex (PFC) $(10,13,15,16)$. Finally, dopamine from a third pathway is synthesized in the hypothalamus and projects to the pituitary gland, where it is involved in the inhibition of prolactin release, a hormone that is important in immune system regulation $^{1}$ (10) (Figure 1). Catecholamines also play an important role in the modulation of the immune system, with dopamine being synthesized and released by immune cells $(17,18)$.

Dopamine receptors (DRs) can be found in both the CNS and in the immune system. There are five types of DRs (D1, D2, D3, D4, and D5), subdivided into two groups: D1-like and D2-like. The D1 and $\mathrm{D} 5$ receptors belong to the D1-like group, while the rest of the DRs belong to the D2-like group of receptors $(10,13)$. These receptors have different distribution densities in the CNS, depending on the brain region. For example, more D1-like receptors are located in the PFC, while more D2-like receptors are found in the striatum. Therefore, different medications have a somewhat specific affinity for DRs and that way can have a greater effect on a specific brain region (e.g., a medication targeting D1 may have more influence on the PFC and its function than on the striatum) (19).

${ }^{1}$ Note that there are actually four pathways: (1) nigrostriatal, (2) mesolimbic, (3) mesocortical, and (4) hypothalamic (tuberoinfundibular). However, in human research, whether it is pharmacological or neuroimaging, it is not possible to specifically delineate the pathways, hence, here, we collapse the second and the third pathways, referring to them as the mesocorticolimbic pathway.

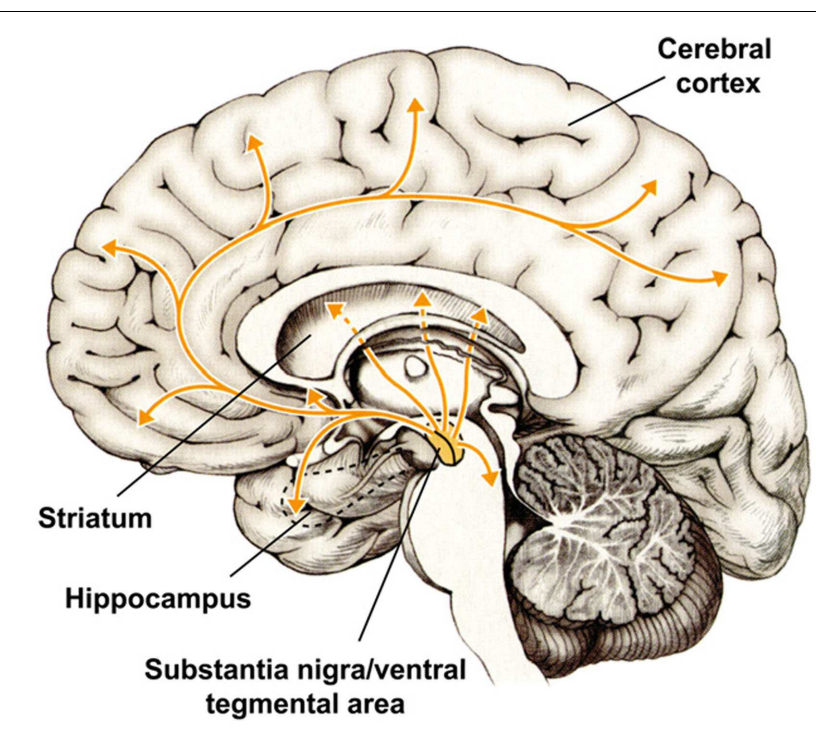

[Dalley, J.W. \& Roiser, J.P. (2012). Neuroscience, 215, 42-58]

FIGURE 1 | A representation of dopaminergic projections throughout the brain. Adapted from Dalley and Roiser (14).
Dopamine has been known to play an important role in motor function. However, evidence from several past decades show that dopamine also plays a significant role in motivation and cognition. Specifically, dopamine has been shown to be involved in learning of action-outcome associations (20-22). In addition, dopamine has been shown to be involved in effortful behavior: the depletion of dopamine from the striatum or the administration of dopamine antagonists has been shown to result in the cessation of effortful reward-seeking behavior. That is, animals that learned to exert effort (e.g., climb a barrier or press a lever several times) for a larger food reward, start to prefer a smaller reward that can be obtained without effort exertion $(23,24)$. PFC dopamine has been shown to play an important role in working memory $(25,26)$. Further, increased amount of dopamine release in the striatum and the PFC has been shown to be associated with cognitive flexibility [see Ref. (27) for review].

\section{THE EFFECTS OF DOPAMINE ON FATIGUE IN THE CENTRAL NERVOUS SYSTEM SUPPORT FROM STRUCTURAL NEUROIMAGING}

Dopamine imbalance can be caused by changes in brain structure, particularly when structures critical for dopaminergic projections are damaged. Recent structural neuroimaging studies implicate regions of the mesocorticolimbic pathway with the fatigue experienced by several clinical populations, including those with MS. Structural impairments in the VMPFC and the striatum have been observed in fatigued individuals, suggesting a role for dopamine in fatigue (28). Pardini et al. (29) found that reduced white matter integrity in the VMPFC, a region that receives dopaminergic projections, was associated with increased fatigue in MS. In another investigation, Pardini et al. (30) assessed fatigue in individuals with TBI, finding that persons with damage localized to the VMPFC had higher levels of fatigue relative to persons with damage localized to the dorsolateral PFC or other areas of the cortex. Genova et al. (31) also showed that individuals with MS who have high fatigue have increased white matter pathology in the internal capsule, which links the striatum with the PFC (32).

Further evidence for the involvement of the mesocorticolimbic regions comes from stroke research: Tang et al. $(33,34)$ reported that striatal infarcts are associated with post-stroke fatigue. Additionally, magnetic resonance spectroscopy findings showed lower levels of choline concentration and $\mathrm{N}$-acetylcholine/creatine ratio (indicative of decreased neuronal integrity) in the striatum in patients with CFS and MS, respectively $(9,35)$.

\section{SUPPORT FROM FUNCTIONAL NEUROIMAGING}

Several functional neuroimaging studies also point to the involvement of mesocorticolimbic pathway in individuals with neurological damage who report fatigue. One of the earliest functional neuroimaging studies that implicated dopaminergic regions in fatigue used positron emission tomography (PET) to assess differences in brain activity at rest (i.e., without task) in individuals with MS (36). MS individuals, who scored high on the Fatigue Severity Scale (FSS) (37) exhibited reduced regional synaptic activity. That is, they exhibited lower levels of glucose metabolism in the PFC and in the striatum compared to individuals with MS who did not report fatigue (36). 
Functional magnetic resonance imaging (fMRI) studies further support the dopamine imbalance hypothesis. Esposito and colleagues (38) examined the influence of fatigue in healthy individuals on resting-state network activity, i.e., task-independent activation of brain networks. Healthy individuals were scanned both at rest and while performing the $n$-back task at the beginning and at the end of: (1) a fatigue-free day and (2) a fatigueinducing day. Participants reported increased mental fatigue and effort after performing the $n$-back task, but only at the end of the fatigue-inducing day. Moreover, reduced connectivity after the fatigue-inducing day was observed in the frontal control network that receives dopaminergic projections and is associated with executive abilities such as working memory. Other recent studies have also found altered connectivity in the mesocorticolimbic pathway in association with fatigue. Engström et al. (39) showed that MS individuals who have high fatigue show reduced mesocorticolimbic connectivity compared to healthy adults during a complex working memory task. Finke et al. (40) showed that high fatigue scores in individuals with MS were negatively correlated with resting-state mesocorticolimbic connectivity. At the same time, pharmacological studies show a reduction in fatigue following a dopamine agonist medication regimen [e.g., Ref. $(41,42)]$. Connectivity between the regions of the mesocorticolimbic pathway has been shown to increase after dopamine agonist administration (bromocriptine and methylphenidate) (43-45). Taken together, these findings suggest that fatigue is associated with reduced connectivity between the regions innervated with dopamine, possibly due to reduced dopamine levels.

A potential difficulty in examining neural correlates of fatigue during task-related functional neuroimaging studies is the assessment of fatigue itself. In most of the clinical studies, fatigue is assessed with a self-report questionnaire, such as the FSS (37) or the Modified Fatigue Impact Scale (MFIS). These questionnaires provide non-specific, "global" data about the effect of "trait" fatigue on physical, social, and other activities performed during the previous weeks. However, functional neuroimaging studies are performed during well-controlled cognitive tasks that are tied to a specific time period and specific cognitive processes. A logical solution for this potential problem is to assess fatigue during task performance. Genova et al. (31) did precisely that: they asked participants with MS to rate their fatigue on a scale from 0 (not at all fatigued) to 100 (most fatigued), before and after a task-switching paradigm, a task that heavily relies on executive processing, during fMRI (46-48). These authors showed that activity in the striatum, a primary input nucleus of the mesocorticolimbic pathway, is greater in MS individuals who had higher on-task, or "state," fatigue compared to healthy individuals (31). Similarly, a recent study reported impaired striatal functioning in individuals with CFS (49). Collectively, these functional neuroimaging findings suggest that individuals with fatigue have impaired functioning of the mesocorticolimbic pathway, likely due to a dopamine imbalance within the regions of this network.

\section{SUPPORT FROM BEHAVIORAL PHARMACOLOGY}

A large body of evidence in support of the dopamine imbalance hypothesis is available from pharmacological studies. Several clinical trials investigated the efficacy of psychostimulant medications on fatigue in MS (50-53). Modafinil is a drug approved for treatment of narcolepsy and has been shown effective in reducing sleepiness (54). This medication might be the drug of choice for fatigue treatment in MS, since fatigue often co-occurs with (or is conflated with) sleepiness (55). However, studies that examined modafinil efficacy for fatigue have been inconclusive due to small sample sizes and methodological issues (open-label) $(2,56,57)$. Amantadine has also been used to treat fatigue in MS. Amantadine is a dopamine agonists that leads to an increase in extracellular dopamine levels through promoting dopamine synthesis and blocking reuptake. A recent randomized blinded trial with four treatment groups (modafinil, amantadine, acetyl-L-carnitine, and placebo) showed amantadine to be successful in reducing fatigue (53). Of note is that Ledinek et al. (53) only included in their study MS individuals who were undergoing interferon-beta treatment (IFN $\beta$ ). IFN $\beta$ is an immunomodulator that recently has been shown to aid in catecholamine synthesis (58), with dopamine being the most common catecholamine, as has been mentioned above. While this evidence is promising, the majority of clinical trials with amantadine are still underpowered and hence cannot provide conclusive evidence (51).

Several recent clinical trials have examined the efficacy of methylphenidate in treating fatigue. Methylphenidate, which has been approved for treatment of attention deficit hyperactivity disorder and narcolepsy, is a dopamine agonist that acts by inhibiting presynaptic dopamine transporters leading to suppression of dopamine reuptake $(59,60)$. That is, due to reuptake suppression, more dopamine remains in the synapse. Recently, a double-blind randomized placebo-controlled (DBRC) study utilizing methylphenidate showed a decrease in fatigue in 36 Parkinson's patients (61). A DBRC trial with 60 CFS patients also showed that $20 \mathrm{mg}$ of methylphenidate over 4 weeks is effective in reducing fatigue (41). Roth et al. (62) evaluated the effectiveness of a $30 \mathrm{mg}$ methylphenidate dose on fatigue in 36 cancer patients in a DBRC trial, resulting in decreased fatigue after 6 weeks of treatment (62). While clinical trials with methylphenidate on MS fatigue are ongoing, the above findings support the dopamine imbalance hypothesis and suggest that restoring dopamine levels by means of dopaminergic medication results in fatigue reduction.

\section{MODULATORY EFFECTS OF DOPAMINE}

Unlike the two major neurotransmitters, glutamate and gamma aminobutyric acid, which have excitatory and inhibitory properties, respectively, dopamine is a neuromodulator. Studies in animals and humans show that the influence of dopamine on cognition follows an inverted " $U$ " shape function $(13,25)$, with too much or too little dopamine administration leading to impaired cognitive performance. Fatigue might be subject to a similar mechanism. In the case of working memory, Gibbs and D'Esposito (63) found that healthy participants who were given a dopamine agonist (i.e., bromocriptine) showed an increase in working memory capacity (63). Harel et al. (64) showed a similar effect in individuals with MS. The authors conducted a DBRC study with 26 MS patients. Patients were classified as working memory impaired according to baseline performance on a complex task that involves working memory, processing speed, and attention. Compared to 
the placebo control group, follow-up task performance in the treatment group significantly improved after a single dose of methylphenidate $(10 \mathrm{mg}$ ) taken $1 \mathrm{~h}$ before task follow-up (64). According to the dopamine imbalance hypothesis, administration of a dopamine agonist, such as the methylphenidate, should have lead to an increase in dopamine levels in the brain and a negative correlation between fatigue and performance; unfortunately, Harel et al. (64) did not report fatigue measures such as the FSS and the MFIS, or on-task fatigue.

Neuroimaging studies on working memory show that performance improvement in individuals with low working memory capacity is accompanied by increased connectivity between mesocorticolimbic structures increases after dopamine agonist administration (43). Vytlacil et al. (65) also showed a correlation between increased connectivity of the striatum and the midbrain nuclei (VTA and SN) and working memory performance after bromocriptine administration in individuals with low working memory capacity; an opposite pattern of results was observed in individuals with high working memory capacity. Similarly to what has been observed in the working memory literature, fatigue has been shown to be associated with reduced connectivity between mesocorticolimbic structures $(39,40)$. However, the effect of dopamine on mesocorticolimbic activation and connectivity in individuals with fatigue has not yet been investigated.

According to the gating hypothesis, dopamine might modulate cognition due to its interaction with other neurotransmitters in the PFC. When there is too much dopamine, the "gate" for excitatory inputs from glutamate neurons to post-synaptic PFC cells shuts down, while too little dopamine allows interference between different inputs $(13,19,25)$. Similar to the gating hypothesis relating dopamine levels to cognition, the dopamine imbalance hypothesis proposes that fatigue might occur when there is too much or too little dopamine. Several studies show that while fatigue decreases with dopaminergic medication, the effect might be dose-dependent. Johansson et al. (42) observed a decrease in fatigue while participants were on a low dose of methylphenidate ( $5 \mathrm{mg}$ ), with an even greater decrease in fatigue observed when participants were on a higher dose of methylphenidate $(20 \mathrm{mg})$. Similar results were obtained in hospice patients (66). Advanced cancer patients who reported high baseline fatigue, had greater fatigue reduction after $20 \mathrm{mg}$ of methylphenidate $(67,68)$. Another DBRC trial with 109 human immunodeficiency virus participants showed methylphenidate titration to be effective in reducing fatigue. However, while some patients took the maximum dose of the medication to achieve fatigue reduction (up to $60 \mathrm{mg}$ per day), other patients were able to achieve fatigue reduction with a lower dose (69).

Taken together, these studies highlight the modulatory effect of dopamine on cognition and fatigue. However, there is no evidence showing an increase in fatigue when there is too much dopamine in the CNS. Therefore, it remains to be tested if and at what dose dopamine medication ceases to be helpful in reducing fatigue. Given that dopamine is a neuromodulator that has been shown to have a variable effect on cognition (i.e., too low or too high levels of dopamine do not improve cognitive functioning), it is likely that it has a similar effect on fatigue. That is, fatigue might result from too much or too little dopamine in the brain.

\section{THE ROLE OF DOPAMINE IN THE IMMUNE SYSTEM}

Based in large part on the evidence from the MS animal model, experimental autoimmune encephalomyelitis (EAE), MS is considered to be an autoimmune disorder of the CNS. To a large extent, the immune system depends on the functioning of the leukocytes or white blood cells. T cells are a type of white blood cell that produce an immune response, i.e., they are activated when the body needs to fight an infection. In autoimmune diseases, including MS, T cells proliferate and attack healthy cells (18), passing though the blood-brain barrier into the CNS. It has been shown that proliferating $\mathrm{CD} 4{ }^{+}$cells (a type of T cells) express the $\mathrm{D} 3$ receptor that contributes to the destruction of dopamine neurons in the SN and generate interferon- $\gamma$, a compound that proliferates inflammation and prevents dopamine synthesis (11, $18,70,71)$. This potentially can result in decreased dopamine levels. Indeed, animal studies showed that CNS dopamine depletion by means of administration of the neurotoxin that kills dopamine cells in the SN leads to EAE exacerbation, while daily administration of a dopamine agonist, bromocriptine that has an affinity for D2 DRs (of which the striatum has a high concentration), has been shown to have beneficial effects on EAE. Treatment with bromocriptine has been shown to result in reduced severity and duration of relapses in rats with acute EAE. It also leads to the suppression of prolactin, a pituitary hormone that is inhibited by dopamine synthesized in the hypothalamus (see above) $(10,11,17$, 72 ). Though highly speculative, this line of reasoning suggests that fatigue might occur due to the dopamine imbalance that starts in the immune system, subsequently affecting the CNS.

IFN $\beta$ is an immunomodulatory drug approved for treatment in relapsing remitting MS. It is the first line of treatment and has been shown to prevent relapses. There are two types of IFN $\beta$ : IFN $\beta-1 \mathrm{a}$ and IFN $\beta-1 \mathrm{~b}$ (73). Even though the precise mechanism of action of the IFN $\beta$ is not completely understood, it is thought that IFN $\beta$ prevents relapses and slows disability progression through retarding inflammatory processes, such as T-cell proliferation and passing of the $\mathrm{CD}^{+}{ }^{+} \mathrm{T}$ cells through the blood-brain barrier. More importantly, recent findings also show that IFN $\beta$ treatment leads to increased production of dopamine $(58,73-75)$.

Given that IFN $\beta$ increases dopamine synthesis, while dopaminergic medications help increase levels of dopamine in the brain, it is possible that individuals with MS on the IFN $\beta$ treatment might benefit more from the dopaminergic fatigue treatment or even have lower levels of fatigue than individuals with MS on a different treatment. To our knowledge, there is only one study that looked at fatigue in relapsing remitting MS with IFN $\beta$ treatment (76). Melanson et al. (76) in a non-randomized open-label study showed that fatigue decreases in patients on IFN $\beta$ treatment.

\section{OTHER FATIGUE HYPOTHESES SEROTONIN}

In their seminal paper, Chaudhuri and Behan (9) called on researchers to clarify the roles of dopamine and serotonin in fatigue. Indeed, both neurotransmitters innervate the basal ganglia, with serotonergic neurons projecting to the basal ganglia from the raphe nuclei (77-79). The serotonin hypothesis developed because fatigue is a symptom of depression that is often treated with selective serotonin reuptake inhibitors. Serotonergic 
levels in the CNS are particularly relevant in sports medicine, as exercise has been shown to increase serotonin levels in the brain, leading to amotivation $(80,81)$.

A few studies in clinical populations provide evidence in support of the serotonin hypothesis, suggesting that increased levels of serotonin might lead to fatigue (82-84). For example, Pavese and colleagues used ${ }^{18} \mathrm{~F}$-DOPA and ${ }^{11} \mathrm{C}-\mathrm{N}, \mathrm{N}$-dimethyl-2-(2-amino4-cyanophenylthio) benzylamine to investigate dopamine storage capacity and serotonin transmission, respectively, in the brain of on-medication PD patients with and without fatigue (85). This PET imaging study specifically focused on the basal ganglia and limbic structures. The region-of-interest analysis revealed significant differences in serotonin transmission between PD patients with and without fatigue. However, significant differences in dopamine storage capacity between PD patients with and without fatigue were revealed only through the voxel-based analysis. Thus, the results of this study support the serotonin hypothesis and suggest that serotonin transmission might play a more important role than striatal dopamine capacity in PD-related fatigue. Unfortunately, the study of Pavese et al. had a rather small sample size (8-10 per group) rendering the results inconclusive.

Clearly, delineating the neurobiological processes underlying such a complex phenomenon as fatigue will not be simple. It is likely that the interaction of several neurotransmitters systems is involved in fatigue. Given the large body of evidence showing the mesocorticolimbic network and, in particular the basal ganglia involvement in fatigue, it is difficult to negate the involvement of serotonin neurons that also innervate the basal ganglia. However, recent studies show that decreased functioning of serotonergic receptors leads to increased functioning of dopaminergic neurons and dopamine release [for review see Ref. (86)]. Therefore, given the preponderance of evidence to date, it appears that the dopamine imbalance hypothesis of fatigue has the most support.

\section{INFLAMMATORY CYTOKINES}

Recently, it has been suggested that inflammatory cytokines, compounds released by the cells of the immune system during inflammation, might be the cause of fatigue. The cytokine hypothesis developed based on the observation that fatigue co-occurs in individuals who have inflammatory biomarkers, such as tumor necrosis factor- $\alpha$, interleukin- 1 , interleukin- 6 , and interferon- $\gamma$ $(8,87-89)$.

A large body of evidence in support of the cytokine hypothesis comes from animal literature $(8,89)$. Indeed, animal studies show that after administration of inflammatory cytokines in the CNS, animals are less willing to exert effort in order to obtain a reward. Nunes et al. (90) showed that administration of the inflammatory cytokine interleukin- $1 \beta$ reduced effortful behavior in rats (decreased amount of lever presses). However, it is important to point out that effortful behavior is dopamine-dependent. Lesioning a region of the fronto-striatal network or dopamine depletion from fronto-striatal regions results in a cessation of effortful behavior $(23,91,92)$. Thus, it is possible that the effect of cytokines on dopamine levels leads to fatigue, with dopamine levels being the culprit in generating fatigue and not the cytokines per se. Indeed, a recent study showed that methamphetamine, a psychostimulant that affects dopamine and, to a lesser extent, serotonin neurons, reduces frontocortical inflammatory cytokine levels (93), while other studies show that inflammatory cytokines have an effect on striatal functioning and dopamine release (87). Thus, such data may support the dopamine imbalance hypothesis, suggesting that the presence of inflammatory cytokines leads to dopamine imbalance.

\section{SUMMARY AND CONCLUSION}

In this review, we propose that fatigue arises due to an imbalance of dopamine, a modulatory neurotransmitter, in the CNS and the immune system. Based on the evidence cited above and building upon a previous framework on fatigue (9), we propose that fatigue depends on the base levels of dopamine in the CNS. Neuroimaging studies in clinical populations with fatigue repeatedly show structural and functional impairments in regions heavily innervated by dopaminergic neurons, namely the striatum and the PFC (See Supplementary Table 1 for the list of studies). While these brain structures underlie a wide range of processes, converging evidence suggests that an imbalance in dopamine plays a key role in fatigue. Indeed, dopaminergic medication that increases dopamine levels in the brain has been shown to increase the functioning and connectivity between these regions in healthy individuals and to decrease fatigue in clinical populations (See Supplementary Table 2 for the list of studies). Thus, the dopamine imbalance hypothesis provides a unifying framework for the study of fatigue.

Given this framework, future research should be geared toward testing specific aspects of the hypothesis. While studies in cognition show that dopamine has a modulatory influence on cognitive performance, clinical trials in fatigue so far only show that dopaminergic medication decreases fatigue. Since fatigue and cognitive functions such as working memory and attention rely on a similar neural network, it is likely that dopamine has a modulatory effect on fatigue as well. Future neuroimaging and pharmacological research is needed to directly test whether this is the case. Thus, an important question is, does fatigue increase as dopamine levels increase above optimal levels? Another question that should be investigated is whether dopamine agonist administration decreases on-task or "state" fatigue in conjunction with performance improvement. This will help in linking objective measures of performance, which have been shown to be affected by dopamine, with subjective on-task fatigue ratings. Pharmacological studies should evaluate the effect of a dopaminergic medication not only in comparison to a placebo but also in comparison with non-dopaminergic medications, to show whether fatigue is differentially affected by a dopaminergic medication versus, for example, serotonergic medication.

Neuroimaging studies should focus on manipulating mesocorticolimbic network activity in controlled experimental settings. This would allow researchers to observe network functioning in fatigued individuals and to answer specific questions about the time course of network activation in a controlled environment. Investigating the time course of network activation during task performance would reveal whether it correlates with on-task fatigue. It is also worth looking at whether the increased connectivity observed after dopamine medication, which has been shown to lead to an increase in working memory performance, is associated with fatigue reduction. Answering these questions will 
provide valuable evidence about the underlying mechanisms of fatigue, and will ultimately allow us to develop targeted treatments for fatigue.

\section{ACKNOWLEDGMENTS}

The authors would like to thank Samantha DePasque Swanson and Angela Spirou for their helpful comments on the manuscript.

\section{SUPPLEMENTARY MATERIAL}

The Supplementary Material for this article can be found online at http://www.frontiersin.org/Journal/10.3389/fneur.2015.00052/ abstract

\section{REFERENCES}

1. Walker LAS, Berard JA, Berrigan LI, Rees LM, Freedman MS. Detecting cognitive fatigue in multiple sclerosis: method matters. J Neurol Sci (2012) 316:86-92. doi:10.1016/j.jns.2012.01.021

2. Zifko UA. Management of fatigue in patients with multiple sclerosis. Drugs (2004) 64:1295-304. doi:10.2165/00003495-200464120-00003

3. Cantor JB, Gordon W, Gumber S. What is post TBI fatigue? NeuroRehabilitation (2013) 32:875-83. doi:10.3233/NRE-130912

4. Friedman JH, Abrantes A, Sweet LH. Fatigue in Parkinson's disease. Expert Opin Pharmacother (2011) 12:1999-2007. doi:10.1517/14656566.2011.587120

5. Barsevick A, Frost M, Zwinderman A, Hall P, Halyard M. I'm so tired: biological and genetic mechanisms of cancer-related fatigue. Qual Life Res (2010) 19:1419-27. doi:10.1007/s11136-010-9757-7

6. Jong E, Oudhoff LA, Epskamp C, Wagener MN, van Duijn M, Fischer S, et al. Predictors and treatment strategies of HIV-related fatigue in the combined antiretroviral therapy era. AIDS (2010) 24:1387-405. doi:10.1097/QAD. 0b013e328339d004

7. Fukuda K, Straus SE, Hickie I, Sharpe MC, Dobbins JG, Komaroff A. The chronic fatigue syndrome: a comprehensive approach to its definition and study. International chronic fatigue syndrome study group. Ann Intern Med (1994) 121:953-9. doi:10.7326/0003-4819-121-12-199412150-00009

8. Harrington ME. Neurobiological studies of fatigue. Prog Neurobiol (2012) 99:93-105. doi:10.1016/j.pneurobio.2012.07.004

9. Chaudhuri A, Behan PO. Fatigue and basal ganglia. J Neurol Sci (2000) 179:34-42. doi:10.1016/S0022-510X(00)00411-1

10. Vallone D, Picetti R, Borrelli E. Structure and function of dopamine receptors. Neurosci Biobehav Rev (2000) 24:125-32. doi:10.1016/S0149-7634(99)00063-9

11. Cosentino M, Marino F. Adrenergic and dopaminergic modulation of immunity in multiple sclerosis: teaching old drugs new tricks? J Neuroimmune Pharmacol (2013) 8:163-79. doi:10.1007/s11481-012-9410-z

12. Nieoullon A. Dopamine and the regulation of cognition and attention. Prog Neurobiol (2002) 67:53-83. doi:10.1016/S0301-0082(02)00011-4

13. Seamans JK, Yang CR. The principal features and mechanisms of dopamine modulation in the prefrontal cortex. Prog Neurobiol (2004) 74:1-57. doi:10. 1016/j.pneurobio.2004.05.006

14. Dalley JW, Roiser JP. Dopamine, serotonin and impulsivity. Neuroscience (2012) 215:42-58. doi:10.1016/j.neuroscience.2012.03.065

15. Haber SN, Fudge JL, McFarland NR. Striatonigrostriatal pathways in primates form an ascending spiral from the shell to the dorsolateral striatum. J Neurosci (2000) 20:2369-82.

16. Haber SN, Kim KS, Mailly P, Calzavara R. Reward-related cortical inputs define a large striatal region in primates that interface with associative cortical connections, providing a substrate for incentive-based learning. J Neurosci (2006) 26:8368-76. doi:10.1523/JNEUROSCI.0271-06.2006

17. Basu S, Dasgupta PS. Dopamine, a neurotransmitter, influences the immune system. J Neuroimmunol (2000) 102:113-24. doi:10.1016/S0165-5728(99)00176-9

18. Pacheco R, Contreras F, Zouali M. The dopaminergic system in autoimmune diseases. Front Immunol (2014) 5:117. doi:10.3389/fimmu.2014.00117

19. Tzschentke TM. Pharmacology and behavioral pharmacology of the mesocortical dopamine system. Prog Neurobiol (2001) 63:241-320. doi:10.1016/S03010082(00)00033-2

20. Hollerman JR, Tremblay L, Schultz W. Influence of reward expectation on behavior-related neuronal activity in primate striatum. J Neurophysiol (1998) 80:947-63.
21. Schultz W. Predictive reward signal of dopamine neurons. J Neurophysiol (1998) 80:1-27.

22. Stefani MR, Moghaddam B. Rule learning and reward contingency are associated with dissociable patterns of dopamine activation in the rat prefrontal cortex, nucleus accumbens, and dorsal striatum. J Neurosci (2006) 26:8810-8. doi:10.1523/JNEUROSCI.1656-06.2006

23. Salamone JD, Correa M, Mingote S, Weber SM. Nucleus accumbens dopamine and the regulation of effort in food-seeking behavior: implications for studies of natural motivation, psychiatry, and drug abuse. J Pharmacol Exp Ther (2003) 305:1-8. doi:10.1124/jpet.102.035063

24. Assadi SM, Yucel M, Pantelis C. Dopamine modulates neural networks involved in effort-based decision-making. Neurosci Biobehav Rev (2009) 33:383-93. doi:10.1016/j.neubiorev.2008.10.010

25. Cools R, D'Esposito M. Inverted-U shaped dopamine actions on human working memory and cognitive control. Biol Psychiatry (2011) 69:e113-25. doi:10.1016/j.biopsych.2011.03.028

26. Takahashi H, Yamada M, Suhara T. Functional significance of central D1 receptors in cognition: beyond working memory. J Cereb Blood Flow Metab (2012) 32:1248-58. doi:10.1038/jcbfm.2011.194

27. Klanker M, Feenstra M, Denys D. Dopaminergic control of cognitive flexibility in humans and animals. Front Neurosci (2013) 7:201. doi:10.3389/ fnins.2013.00201

28. Dobryakova E, DeLuca J, Genova HM, Wylie GR. Neural correlates of cognitive fatigue: cortico-striatal circuitry and effort-reward imbalance. J Int Neuropsych Soc (2013) 19(8):849-53. doi:10.1017/S1355617713000684

29. Pardini M, Bonzano L, Mancardi GL, Roccatagliata L. Frontal networks play a role in fatigue perception in multiple sclerosis. Behav Neurosci (2010) 124:329-36. doi:10.1037/a0019585

30. Pardini M, Krueger F, Raymont V, Grafman J. Ventromedial prefrontal cortex modulates fatigue after penetrating traumatic brain injury. Neurology (2010) 74:749-54. doi:10.1212/WNL.0b013e3181d25b6b

31. Genova HM, Rajagopalan V, Deluca J, Das A, Binder A, Arjunan A, et al. Examination of cognitive fatigue in multiple sclerosis using functional magnetic resonance imaging and diffusion tensor imaging. PLoS One (2013) 8:e78811. doi:10.1371/journal.pone.0078811

32. Koch K, Wagner G, Schachtzabel C, Schultz CC, Güllmar D, Reichenbach JR, et al. Association between white matter fiber structure and reward-related reactivity of the ventral striatum. Hum Brain Mapp (2013) 35(4):1469-76. doi:10.1002/hbm.22284

33. Tang WK, Chen YK, Mok V, Chu WCW, Ungvari GS, Ahuja AT, et al. Acute basal ganglia infarcts in poststroke fatigue: an MRI study. J Neurol (2010) 257:178-82. doi:10.1007/s00415-009-5284-2

34. Tang WK, Liang HJ, Chen YK, Chu WCW, Abrigo J, Mok VCT, et al. Poststroke fatigue is associated with caudate infarcts. J Neurol Sci (2013) 15:131-5. doi:10.1016/j.jns.2012.10.022

35. Téllez N, Alonso J, Río J, Tintoré M, Nos C, Montalban X, et al. The basal ganglia: a substrate for fatigue in multiple sclerosis. Neuroradiology (2008) 50:17-23. doi:10.1007/s00234-007-0304-3

36. Roelcke U, Kappos L, Lechner-Scott J, Brunnschweiler H, Huber S, Ammann $\mathrm{W}$, et al. Reduced glucose metabolism in the frontal cortex and basal ganglia of multiple sclerosis patients with fatigue: a $18 \mathrm{~F}-$ fluorodeoxyglucose positron emission tomography study. Neurology (1997) 48:1566-71. doi:10.1212/WNL. 48.6.1566

37. Krupp LB, LaRocca NG, Muir-Nash J, Steinberg AD. The fatigue severity scale. Application to patients with multiple sclerosis and systemic lupus erythematosus. Arch Neurol (1989) 46:1121-3. doi:10.1001/archneur.1989. 00520460115022

38. Esposito F, Otto T, Zijlstra FRH, Goebel R. Spatially distributed effects of mental exhaustion on resting-state FMRI networks. PloS One (2014) 9(4):e94222. doi:10.1371/journal.pone.0094222

39. Engström M, Flensner G, Landtblom A-M, Ek A-C, Karlsson T. Thalamostriato-cortical determinants to fatigue in multiple sclerosis. Brain Behav (2013) 3:715-28. doi:10.1002/brb3.181

40. Finke C, Schlichting J, Papazoglou S, Scheel M, Freing A, Soemmer C, et al. Altered basal ganglia functional connectivity in multiple sclerosis patients with fatigue. Mult Scler (2014). doi:10.1177/1352458514555784

41. Blockmans D, Persoons P, Van Houdenhove B, Bobbaers H. Does methylphenidate reduce the symptoms of chronic fatigue syndrome? Am J Med (2006) 119:e23-30. doi:10.1016/j.amjmed.2005.07.047 
42. Johansson B, Wentzel A, Andrell P, Odenstedt J, Mannheimer C, Ronnback L. Evaluation of dosage, safety and effects of methylphenidate on post-traumatic brain injury symptoms with a focus on mental fatigue and pain. Brain Inj (2014) 28:304-10. doi:10.3109/02699052.2013.865267

43. Wallace DL, Vytlacil JJ, Nomura EM, Gibbs SEB, D'Esposito M. The dopamine agonist bromocriptine differentially affects fronto-striatal functional connectivity during working memory. Front Hum Neurosci (2011) 5:32. doi:10.3389/ fnhum.2011.00032

44. Konova AB, Moeller SJ, Tomasi D, Volkow ND, Goldstein RZ. Effects of methylphenidate on resting-state functional connectivity of the mesocorticolimbic dopamine pathways in cocaine addiction. JAMA Psychiatry (2013) 70:857-68. doi:10.1001/jamapsychiatry.2013.1129

45. Mueller S, Costa A, Keeser D, Pogarell O, Berman A, Coates U, et al. The effects of methylphenidate on whole brain intrinsic functional connectivity. Hum Brain Mapp (2014) 35(11):5379-88. doi:10.1002/hbm.22557

46. Wylie GR, Javitt DC, Foxe JJ. Jumping the gun: is effective preparation contingent upon anticipatory activation in task-relevant neural circuitry? Cereb Cortex (2006) 16:394-404. doi:10.1093/cercor/bhi118

47. Wylie GR, Sumowski JF, Murray M. Are there control processes, and (if so) can they be studied? Psychol Res (2011) 75(6):535-43. doi:10.1007/s00426-0110354-3

48. Weaver SM, Foxe JJ, Shpaner M, Wylie GR. You can't always get what you want: the influence of unexpected task constraint on voluntary task switching. Q J Exp Psychol (Hove) (2014) 67(11):2247-59. doi:10.1080/17470218.2014.917115

49. Miller AH, Jones JF, Drake DF, Tian H, Unger ER, Pagnoni G. Decreased basal ganglia activation in subjects with chronic fatigue syndrome: association with symptoms of fatigue. PLoS One (2014) 9:e98156. doi:10.1371/journal.pone. 0098156

50. Krupp LB, Coyle PK, Doscher C, Miller A, Cross AH, Jandorf L, et al. Fatigue therapy in multiple sclerosis: results of a double-blind, randomized, parallel trial of amantadine, pemoline, and placebo. Neurology (1995) 45:1956-61. doi:10.1212/WNL.45.11.1956

51. Pucci E, Branãs P, D’Amico R, Giuliani G, Solari A, Taus C. Amantadine for fatigue in multiple sclerosis. Cochrane Database Syst Rev (2007) 1:CD002818. doi:10.1002/14651858.CD002818.pub2

52. Stankoff B, Waubant E, Confavreux C, Edan G, Debouverie M, Rumbach L, et al. Modafinil for fatigue in MS: a randomized placebo-controlled doubleblind study. Neurology (2005) 64:1139-43. doi:10.1212/01.WNL.0000158272. $27070.6 \mathrm{~A}$

53. Ledinek AH, Sajko MC, Rot U. Evaluating the effects of amantadine, modafinil and acetyl-L-carnitine on fatigue in multiple sclerosis - result of a pilot randomized, blind study. Clin Neurol Neurosurg (2013) 1155:S86-9. doi:10.1016/j. clineuro.2013.09.029

54. Kumar R. Approved and investigational uses of modafinil: an evidence-based review. Drugs (2008) 68:1803-39. doi:10.2165/00003495-200868130-00003

55. Kaminska M, Kimoff RJ, Schwartzman K, Trojan DA. Sleep disorders and fatigue in multiple sclerosis: evidence for association and interaction. J Neurol Sci (2011) 302:7-13. doi:10.1016/j.jns.2010.12.008

56. Rammohan KW, Rosenberg JH, Lynn DJ, Blumenfeld AM, Pollak CP, Nagaraja HN. Efficacy and safety of modafinil (provigil) for the treatment of fatigue in multiple sclerosis: a two centre phase 2 study. J Neurol Neurosurg Psychiatry (2002) 72:179-83. doi:10.1136/jnnp.72.2.179

57. Sheng P, Hou L, Wang X, Wang X, Huang C, Yu M, et al. Efficacy of modafinil on fatigue and excessive daytime sleepiness associated with neurological disorders: a systematic review and meta-analysis. PLoS One (2013) 8:e81802. doi:10.1371/journal.pone.0081802

58. Zaffaroni M, Marino F, Bombelli R, Rasini E, Monti M, Ferrari M, et al. Therapy with interferon-beta modulates endogenous catecholamines in lymphocytes of patients with multiple sclerosis. Exp Neurol (2008) 214:315-21. doi:10.1016/j.expneurol.2008.08.015

59. Volkow ND, Wang G, Fowler JS, Logan J, Gerasimov M, Maynard L, et al. Therapeutic doses of oral methylphenidate significantly increase extracellular dopamine in the human brain. J Neurosci (2001) 21:RC121.

60. Prommer E. Methylphenidate: established and expanding roles in symptom management. Am J Hosp Palliat Care (2012) 29:483-90. doi:10.1177/ 1049909111427029

61. Mendonça DA, Menezes K, Jog MS. Methylphenidate improves fatigue scores in Parkinson disease: a randomized controlled trial. Mov Disord (2007) 22:2070-6. doi: $10.1002 /$ mds. 21656
62. Roth AJ, Nelson C, Rosenfeld B, Scher H, Slovin S, Morris M, et al. Methylphenidate for fatigue in ambulatory men with prostate cancer. Cancer (2010) 116:5102-10. doi:10.1002/cncr.25424

63. Gibbs SEB, D’Esposito M. Individual capacity differences predict working memory performance and prefrontal activity following dopamine receptor stimulation. Cogn Affect Behav Neurosci (2005) 5:212-21. doi:10.3758/CABN.5.2.212

64. Harel Y, Appleboim N, Lavie M, Achiron A. Single dose of methylphenidate improves cognitive performance in multiple sclerosis patients with impaired attention process. J Neurol Sci (2009) 276:38-40. doi:10.1016/j.jns. 2008.08.025

65. Vytlacil J, Kayser A, Miyakawa A, D’Esposito M. An approach for identifying brainstem dopaminergic pathways using resting state functional MRI. PLoS One (2014) 9:e87109. doi:10.1371/journal.pone.0087109

66. Kerr CW, Drake J, Milch RA, Brazeau DA, Skretny JA, Brazeau GA, et al. Effects of methylphenidate on fatigue and depression: a randomized, doubleblind, placebo-controlled trial. J Pain Symptom Manage (2012) 43:68-77. doi:10.1016/j.jpainsymman.2011.03.026

67. Moraska AR, Sood A, Dakhil SR, Sloan JA, Barton D, Atherton PJ, et al. Phase III, randomized, double-blind, placebo-controlled study of long-acting methylphenidate for cancer-related fatigue: north central cancer treatment group NCCTG-N05C7 trial. J Clin Oncol (2010) 28:3673-9. doi:10.1200/JCO. 2010.28.1444

68. Yennurajalingam S, Palmer JL, Chacko R, Bruera E. Factors associated with response to methylphenidate in advanced cancer patients. Oncologist (2011) 16:246-53. doi:10.1634/theoncologist.2010-0214

69. Breitbart W, Rosenfeld B, Kaim M, Funesti-Esch J. A randomized, double-blind, placebo-controlled trial of psychostimulants for the treatment of fatigue in ambulatory patients with human immunodeficiency virus disease. Arch Intern Med (2001) 161:411-20. doi:10.1001/archinte.161.3.411

70. Cosentino M, Zaffaroni M, Ferrari M, Marino F, Bombelli R, Rasini E, et al. Interferon-gamma and interferon-beta affect endogenous catecholamines in human peripheral blood mononuclear cells: implications for multiple sclerosis. J Neuroimmunol (2005) 162:112-21. doi:10.1016/j.jneuroim.2005.01.019

71. González H, Contreras F, Prado C, Elgueta D, Franz D, Bernales S, et al. Dopamine receptor D3 expressed on CD4+ T cells favors neurodegeneration of dopaminergic neurons during Parkinson's disease. J Immunol (2013) 190:5048-56. doi:10.4049/jimmunol.1203121

72. Dijkstra CD, van der Voort ER, De Groot CJA, Huitinga I, Uitdehaag BMJ, Polman $\mathrm{CH}$, et al. Therapeutic effect of the D2-dopamine agonist bromocriptine on acute and relapsing experimental allergic encephalomyelitis. Psychoneuroendocrinology (1994) 19:135-42. doi:10.1016/0306-4530(94)90003-5

73. Dhib-Jalbut S, Marks S. Interferon-beta mechanisms of action in multiple sclerosis. Neurol (2010) 74(Suppl 1):S17-24. doi:10.1212/WNL.0b013e3181c97d99

74. Giorelli M, Livrea P, Trojano M. Dopamine fails to regulate activation of peripheral blood lymphocytes from multiple sclerosis patients: effects of IFN-beta. J Interferon Cytokine Res (2005) 25:395-406. doi:10.1089/jir.2005.25.395

75. Cosentino M, Zaffaroni M, Trojano M, Giorelli M, Pica C, Rasini E, et al. Dopaminergic modulation of CD4+CD25(high) regulatory $\mathrm{T}$ lymphocytes in multiple sclerosis patients during interferon- $\beta$ therapy. Neuroimmunomodulation (2012) 19:283-92. doi:10.1159/000336981

76. Melanson M, Grossberndt A, Klowak M, Leong C, Frost EE, Prout M, et al. Fatigue and cognition in patients with relapsing multiple sclerosis treated with interferon B. Int J Neurosci (2010) 120:631-40. doi:10.3109/00207454.2010. 511732

77. Parent M, Wallman M-J, Gagnon D, Parent A. Serotonin innervation of basal ganglia in monkeys and humans. J Chem Neuroanat (2011) 41:256-65. doi:10.1016/j.jchemneu.2011.04.005

78. Mathur BN, Lovinger DM. Serotonergic action on dorsal striatal function. Parkinsonism Relat Disord (2012) 1:129-31. doi:10.1016/S1353-8020(11) 70040-2

79. De Deurwaerdère $P$, Lagière $M$, Bosc $M$, Navailles $S$. Multiple controls exerted by 5-HT2C receptors upon basal ganglia function: from physiology to pathophysiology. Exp Brain Res (2013) 230:477-511. doi:10.1007/s00221-013-3508-2

80. Meeusen R, Watson P, Hasegawa H, Roelands B, Piacentini MF. The serotonin hypothesis and beyond. Sports Med (2006) 36:881-909. doi:10.2165/00007256200636100-00006

81. Meeusen R, Roelands B. Central fatigue and neurotransmitters, can thermoregulation be manipulated? Scand J Med Sci Sports (2010) 20(Suppl 3):19-28. doi:10.1111/j.1600-0838.2010.01205.x 
82. Yamamoto S, Ouchi Y, Onoe H, Yoshikawa E, Tsukada H, Takahashi H, et al. Reduction of serotonin transporters of patients with chronic fatigue syndrome. Neuroreport (2004) 15:2571-4. doi:10.1097/00001756-20041203000002

83. Hesse S, Moeller F, Petroff D, Lobsien D, Luthardt J, Regenthal R, et al. Altered serotonin transporter availability in patients with multiple sclerosis. Eur J Nucl Med Mol Imaging (2014) 41:827-35. doi:10.1007/s00259-013-2636-z

84. The GKH, Verkes RJ, Fekkes D, Bleijenberg G, van der Meer JWM, Buitelaar JK. Tryptophan depletion in chronic fatigue syndrome, a pilot cross-over study. BMC Res Notes (2014) 7:650. doi:10.1186/1756-0500-7-650

85. Pavese N, Metta V, Bose SK, Chaudhuri KR, Brooks DJ. Fatigue in Parkinson's disease is linked to striatal and limbic serotonergic dysfunction. Brain (2010) 133:3434-43. doi:10.1093/brain/awq268

86. Di Giovanni G, Esposito E, Di Matteo V. Role of serotonin in central dopamine dysfunction. CNS Neurosci Ther (2010) 16:179-94. doi:10.1111/j.1755-5949. 2010.00135.x

87. Felger JC, Miller AH. Cytokine effects on the basal ganglia and dopamine function: the subcortical source of inflammatory malaise. Front Neuroendocrinol (2012) 33:315-27. doi:10.1016/j.yfrne.2012.09.003

88. Smith AP. Twenty-five years of research on the behavioural malaise associated with influenza and the common cold. Psychoneuroendocrinology (2013) 38:744-51. doi:10.1016/j.psyneuen.2012.09.002

89. Dantzer R, Heijnen CJ, Kavelaars A, Laye S, Capuron L. The neuroimmune basis of fatigue. Trends Neurosci (2014) 37:39-46. doi:10.1016/j.tins.2013.10.003

90. Nunes EJ, Randall PA, Estrada A, Epling B, Hart EE, Lee CA, et al. Effort-related motivational effects of the pro-inflammatory cytokine interleukin 1-beta: studies with the concurrent fixed ratio 5/ chow feeding choice task. Psychopharmacology (Berl) (2014) 231:727-36. doi:10.1007/s00213-0133285-4
91. Salamone JD, Correa M, Nunes EJ, Randall PA, Pardo M. The behavioral pharmacology of effort-related choice behavior: dopamine, adenosine and beyond. J Exp Anal Behav (2012) 97:125-46. doi:10.1901/jeab.2012.97-125

92. Ishiwari K, Weber SM, Mingote S, Correa M, Salamone JD. Accumbens dopamine and the regulation of effort in food-seeking behavior: modulation of work output by different ratio or force requirements. Behav Brain Res (2004) 151:83-91. doi:10.1016/j.bbr.2003.08.007

93. Stolyarova A, Thompson AB, Barrientos RM, Izquierdo A. Reductions in frontocortical cytokine levels are associated with long-lasting alterations in reward valuation after methamphetamine. Neuropsychopharmacology (2014). doi:10.1038/npp.2014.309

Conflict of Interest Statement: The authors declare that the research was conducted in the absence of any commercial or financial relationships that could be construed as a potential conflict of interest.

Received: 29 October 2014; accepted: 25 February 2015; published online: 12 March 2015.

Citation: Dobryakova E, Genova HM, DeLuca J and Wylie GR (2015) The dopamine imbalance hypothesis of fatigue in multiple sclerosis and other neurological disorders. Front. Neurol. 6:52. doi: 10.3389/fneur.2015.00052

This article was submitted to Multiple Sclerosis and Neuroimmunology, a section of the journal Frontiers in Neurology.

Copyright (c) 2015 Dobryakova, Genova, DeLuca and Wylie. This is an open-access article distributed under the terms of the Creative Commons Attribution License (CC $B Y)$. The use, distribution or reproduction in other forums is permitted, provided the original author(s) or licensor are credited and that the original publication in this journal is cited, in accordance with accepted academic practice. No use, distribution or reproduction is permitted which does not comply with these terms. 